\title{
GRADED LIE ALGEBRAS WITH FINITE POLYDEPTH
}

\author{
By Yves FELIX, Stephen HALPERIN And JeAn-Claude THOMAS
}

ABSTRACT. - If $A$ is a graded connected algebra then we define a new invariant, polydepth $A$, which is finite if $\operatorname{Ext}_{A}^{*}(M, A) \neq 0$ for some $A$-module $M$ of at most polynomial growth. THEOREM 1: If $f: X \rightarrow Y$ is a continuous map of finite category, and if the orbits of $H_{*}(\Omega Y)$ acting in the homology of the homotopy fibre grow at most polynomially, then $H_{*}(\Omega Y)$ has finite polydepth. THEOREM 5: If $L$ is a graded Lie algebra and polydepth $U L$ is finite then either $L$ is solvable and $U L$ grows at most polynomially or else for some integer $d$ and all $r, \sum_{i=k+1}^{k+d} \operatorname{dim} L_{i} \geqslant k^{r}, k \geqslant$ some $k(r)$.

(C) 2003 Elsevier SAS

RÉSUMÉ. - Si $A$ est une algèbre graduée connexe nous définissons un nouvel invariant, appelé la profondeur polynomiale noté polydepth $A$, qui est fini s'il existe un $A$-module gradué $M$ ayant une croissance au plus polynomiale tel que $\operatorname{Ext}_{A}^{*}(M, A) \neq 0$. THÉORÈmE $1:$ si $f: X \rightarrow Y$ est une application continue de LS-catégorie finie et si les orbites de l'action de $H_{*}(\Omega Y)$ sur l'homologie de la fibre homotopique de $f$ possèdent une croissance au plus polynomiale alors polydepth $H_{*}(\Omega Y)$ est finie. THÉORÈME 5 : si $L$ est une algèbre de Lie graduée et si polydepth $U L$ est fini alors, soit $L$ est résoluble et $U L$ possède une croissance au plus polynomiale, soit il existe un entier $d$ tel que pour tout entier $r$ on ait $\sum_{i=k+1}^{k+d} \operatorname{dim} L_{i} \geqslant k^{r}$ pour tous les $k$ plus grands qu'un certain $k(r)$.

(c) 2003 Elsevier SAS

We work over a field $l k$ of characteristic different from 2. If $V=\left\{V_{k}\right\}$ is a graded vector space we denote by $V^{\#}=\left\{\operatorname{Hom}_{l k}\left(V_{k}, l k\right)\right\}$ the dual graded vector space. A graded Lie algebra is a graded vector space $L$, equipped with a bilinear map [, ]: $L_{i} \times L_{j} \rightarrow L_{i+j}$ satisfying

$$
[x, y]+(-1)^{i j}[y, x]=0
$$

and

$$
[x,[y, z]]=[[x, y], z]+(-1)^{i j}[y,[x, z]]
$$

for $x \in L_{i}, y \in L_{j}, z \in L$. It follows that $3[x,[x, x]]=0$ for $x$ of odd degree, and so if char $k=3$ we further require that $[x,[x, x]]=0$. Finally we consider only graded Lie algebras satisfying $L=\left\{L_{i}\right\}_{i \geqslant 1}$ and each $L_{i}$ is finite dimensional. (Any graded vector space $V$ with each $V_{i}$ finite dimensional is said to have finite type.)

The universal enveloping algebra of $L$ is denoted by $U L$ and it satisfies the classical PoincaréBirkhoff-Witt Theorem (in characteristic 3 this uses the $[x,[x, x]]=0$ requirement).

Important examples appear in topology. Let $X$ be a simply connected topological space with rational homology of finite type. Then the rational homotopy Lie algebra $L_{X}$ of $X$ is defined by

$$
L_{X}=\pi_{*}(\Omega X) \otimes \mathbb{Q} ; \quad[,]=\text { Samelson product }
$$


and the Hurewicz map extends to an isomorphism, [10],

$$
U L_{X} \stackrel{\cong}{\longrightarrow} H_{*}(\Omega X ; \mathbb{Q}) \text {. }
$$

Analogously, if $X$ is a finite $n$-dimensional and $r$-connected CW complex, then for primes $p>n / r, H_{*}\left(\Omega X ; \mathbb{F}_{p}\right)=U E$ for some graded Lie algebra $E$ [8].

If $M$ is a module over a (graded) algebra $A$ then the grade of $M$, grade $M$, is the least integer $q($ or $\infty)$ such that $\operatorname{Ext}_{A}^{q}(M, A) \neq 0$. And if $V=\left\{V_{i}\right\}_{i \geqslant 0}$ is a graded vector space then $V$ has at most polynomial growth if for some constant $C$, and some non-negative integer, $d$, $\sum_{i \leqslant n} \operatorname{dim} V_{i} \leqslant C n^{d}, n \geqslant 1$. In this case the least such $d$ is called the polynomial bound for the growth of $V$ and is denoted by polybd $(V)$. If $V$ does not have at most polynomial growth we put polybd $V=\infty$ and we say that $V$ grows faster than any polynomial.

In this paper we combine these two notions in the

Definition. - The polygrade of an $A$-module, $M$, is the sum, grade $M+\operatorname{polybd} M$, and the polydepth of $A$ is the least integer (or $\infty$ ) occurring as the polygrade of an $A$-module.

In the case $A=U L$ the unique augmentation $U L \rightarrow l k$ makes $l k$ into a $U L$-module, and by definition, the grade of $l k$ is the depth of $U L$. Since polybd $l k=0$ it follows that:

$$
\text { polydepth } U L \leqslant \operatorname{depth} U L \text {. }
$$

Moreover (cf. Proposition 1.6) if $\operatorname{dim} L<\infty$ then equality holds. We shall abuse notation and refer to these invariants respectively as polydepth $L$ and depth $L$.

Note that $\operatorname{Ext}_{U L}^{0}(U L, U L)$ contains the identity map and so

$$
\text { polydepth } L \leqslant \operatorname{polybd} U L \text {. }
$$

Observe as well that for any graded vector space $M$, polybd $M=0$ if and only if $\operatorname{dim} M$ is finite. Thus polydepth $L=0$ if and only if depth $L=0$, which happens if and only if $L$ is finite dimensional and concentrated in odd degrees.

Depth has been a useful concept in topology because, on the one hand, LusternikSchnirelmann category satisfies [1]

$$
\operatorname{depth} L_{X} \leqslant \operatorname{cat} X
$$

and, on the other hand [3-5], finite depth has important implications for the structure of a graded Lie algebra.

The purpose of this paper is to show that essentially the same implications follow from the weaker hypothesis that polydepth $L$ is finite, while simultaneously identifying a larger class of topological spaces and Lie algebras for which the weaker hypothesis holds.

Indeed, we have

THEOREM 1. - If $F \rightarrow X \stackrel{f}{\longrightarrow} Y$ is a fibration of path-connected spaces, then

$$
\text { polydepth } H_{*}(\Omega Y) \leqslant \operatorname{polybd} H_{*}(F)+\operatorname{cat} f \text {. }
$$

Proof. - The fibration determines an action up to homotopy of $\Omega Y$ on $F$, which makes $H_{*}(F)$ into an $H_{*}(\Omega Y)$-module. According to [6], grade $H_{*}(F) \leqslant$ cat $f$.

Our main structural theorems read: 
THEOREM 2. - Let $E(L)$ denote the linear span of elements $x \in L_{\text {even }}$ such that ad $x$ acts nilpotently on each $y \in L$. Then

$$
\operatorname{dim} E(L) \leqslant \operatorname{polydepth} L
$$

THEOREM 3. - The following conditions on a graded Lie algebra L are equivalent

(i) $L$ is the union of solvable ideals and polydepth $L$ is finite;

(ii) $U L$ grows at most polynomially (polybd $U L$ is finite);

(iii) $L_{\text {even }}$ is finite dimensional, and for some constant $C$

$$
\sum_{i \leqslant n} \operatorname{dim} L_{i} \leqslant C \log _{2} n, \quad n \geqslant 1
$$

In this case $L$ is solvable.

THEOREM 4. - If L is a graded Lie algebra of finite polydepth then the union of the solvable ideals of $L$ is a solvable ideal of finite polydepth.

THEOREM 5. - Suppose polydepth $L$ is finite and $L$ is not solvable. Then there is an integer $d$ such that for all $r \geqslant 1$ :

$$
\sum_{i=k+1}^{k+d} \operatorname{dim} L_{i} \geqslant k^{r}, \quad k \geqslant \text { some } k(r) .
$$

Remark. - In [7] it is shown that if $L=L_{X}$ where $X$ is a finite 1-connected CW complex, then we may take $d=\operatorname{dim} X$ in Theorem 5 .

\section{Properties of polydepth}

LEMMA 1.1.-If $M$ is a module for some graded algebra $A$ of finite type and if $\operatorname{Ext}_{A}^{q}(M, A) \neq 0$ then $\operatorname{Ext}_{A}^{q}(A \cdot x, A) \neq 0$ for some $x$ in a subquotient module of $M$.

Proof. - Recall that $A^{\#}=\operatorname{Hom}_{l k}(A, l k)$. Then $\operatorname{Ext}_{A}^{q}(M, A)$ is the dual of $\operatorname{Tor}_{q}^{A}\left(M, A^{\#}\right)$ and a direct limit argument shows that for some $x_{1}, \ldots, x_{n} \in M$,

$$
\operatorname{Tor}_{q}^{A}\left(A \cdot x_{1}+\cdots+A \cdot x_{n}, A^{\#}\right) \neq 0 .
$$

Now use the exact sequence associated to the inclusion

$$
A \cdot x_{1}+\cdots+A \cdot x_{n-1} \in A \cdot x_{1}+\cdots+A \cdot x_{n} .
$$

Corollary 1.2. - Polydepth $A$ is the least $m$ such that polygrade $N=m$ for some monogenic A-module $N$.

Remark. - It follows from the Corollary that we may improve Theorem 1 to the inequality

$$
\text { polydepth } H_{*}(\Omega Y) \leqslant \operatorname{polybd}\left(H_{*}(\Omega Y) \cdot \alpha\right)+\operatorname{cat} f, \quad \text { some } \alpha \in H_{*}(F) .
$$

PROPOSITION 1.3. - Let L be a graded Lie algebra.

(i) Each ideal satisfies polydepth $I \leqslant \operatorname{polydepth} L$. 
(ii) Let $E$ be a Lie subalgebra of $L$. If $L$ has finite polydepth and if for each $x \in L / E$ the orbit $U E \cdot x$ has at most polynomial growth, then $E$ has finite polydepth.

(iii) For $n$ sufficiently large the sub Lie algebra E generated by $L_{\leqslant n}$ satisfies

$$
\text { polydepth } E \leqslant \text { polydepth } L \text {. }
$$

Proof. - (i) This follows from the Hochschild-Serre spectral sequence, converging from $\operatorname{Ext}_{U L / I}^{p}\left(l k, \operatorname{Ext}_{U I}^{q}(M, U L)\right)$ to $\operatorname{Ext}_{U L}^{p+q}(M, U L)$. (Note that since $U L$ is $U I$-free, $\operatorname{grade}_{U I}(M)$ is the least $q$ such that $\operatorname{Ext}_{U I}^{q}(M, U L) \neq 0$.)

(ii) As in Lemma 1.1, $\operatorname{Ext}_{U L}^{q}(M, U L)$ is dual to $\operatorname{Tor}_{q}^{U L}\left(M,(U L)^{\#}\right)$, and this is the homology of the Cartan-Eilenberg-Chevalley complex $\wedge s L \otimes M \otimes(U L)^{\#}$. Write $L=E \oplus V$ and set $F_{p}=\wedge s E \otimes \wedge \leqslant p s V \otimes M \otimes(U L)^{\#}$. This filtration determines a convergent spectral sequence, introduced by Koszul in [9], and which is the Hochschild-Serre spectral sequence when $E$ is an ideal. The $E^{1}$-term of the spectral sequence is $\operatorname{Tor}_{q}^{U E}\left(\wedge^{p} s L / E \otimes M,(U L)^{\#}\right)$, converging to $\operatorname{Tor}_{p+q}^{U L}\left(M,(U L)^{\#}\right)$.

Each element $z \in \wedge^{p} s L / E \otimes M$ is contained in a finite sum of $U E$-modules of the form $s\left(U E \cdot x_{1}\right) \wedge \cdots \wedge s\left(U E \cdot x_{p}\right) \otimes M$ and it follows that

$$
\operatorname{polybd}(U E \cdot z) \leqslant p \cdot \operatorname{polybd}(U E \cdot x)+\operatorname{polybd}(M)
$$

for some $x \in L / E$. Choose $M$ so that polydepth $L=$ polygrade $M$ and apply Lemma 1.1 with $p+q=\operatorname{grade} M$.

(iii) If $\operatorname{Ext}_{U L}^{p}(M, U L)$ is non-zero and $\operatorname{polybd}(M)<\infty$ it suffices to choose $E$ so that the restriction $\operatorname{Ext}_{U L}^{p}(M, U L) \rightarrow \operatorname{Ext}_{U E}^{p}(M, U L)$ is non-zero ([4], Proposition 3.1).

COROLlaRY 1.4 (of the proof of (ii)). - Suppose for some $k \geqslant 1$ that $\operatorname{polybd}(U E \cdot x) \leqslant k$, $x \in L / E$. Then polydepth $E \leqslant k$ polydepth $L$.

COROLLARY 1.5. - Let $E$ be a sub-Lie algebra of a graded Lie algebra L. If L has finite polydepth and $L / E$ has at most polynomial growth, then $E$ has finite polydepth.

Example 1. - Let $\mathbb{L}(V)$ be the free Lie algebra on a graded vector space $V$. Then for any graded Lie algebra $L, L \coprod \mathbb{L}(V)$ has depth 1 . Thus the injection $L \rightarrow L \coprod \mathbb{L}(V)$ shows that each graded Lie algebra is a sub-Lie algebra of a Lie algebra of finite polydepth. The previous corollary gives restriction on a Lie algebra $L$ for being a sub-Lie algebra of a Lie algebra of finite polydepth, $K$, when the quotient has at most polynomial growth.

Proposition 1.6. - If $L$ is a finite dimensional graded Lie algebra then

$$
\text { polydepth } L=\operatorname{depth} L \text {. }
$$

Proof. - As observed in the introduction, polydepth $L \leqslant \operatorname{depth} L$. On the other hand, by Lemma 1.1 , polydepth $L=$ polygrade $M$ for some monogenic module $M=U L \cdot x$. Now Theorem 3.1 in [3] asserts that polygrade $M=\operatorname{depth} L$.

\section{Proof of Theorem 2}

Suppose $I \subset L$ is an ideal. If $\operatorname{Ext}_{U L}^{m}(M, U L) \neq 0$, then $\operatorname{Ext}_{U L / I}^{p}\left(\operatorname{Tor}_{q}^{U I}(M, k), U L / I\right) \neq 0$, some $p+q=m$. (Same proof as in: [3], Lemma 4.3, for the case $M=l k$.) By Lemma 1.1 there is a monogenic $U L / I$-module $N$ such that $N$ is a subquotient of $\operatorname{Tor}_{q}^{U I}(M, l k)$, and grade $N \leqslant p$. 
Now suppose $L / I$ is finite dimensional. Then Theorem 3.1 in [3] asserts that

$$
\operatorname{grade} N+\operatorname{polybd} N=\operatorname{dim}(L / I)_{\text {even }}
$$

On the other hand, write $(L / I)_{\text {even }}=V \oplus W$ where $V$ is the image of $E(L)$. Let $x_{i} \in L_{\text {even, }}$, $y_{j} \in L_{\text {odd }}$ and $z_{k} \in E(L)$ represent respectively bases of $W,(L / I)_{\text {odd }}$ and $V$. Then the elements $x_{1}^{k_{1}} \cdots x_{s}^{k_{s}} y_{1}^{\varepsilon_{1}} \cdots y_{t}^{\varepsilon_{t}} z_{1}^{m_{1}} \cdots z_{u}^{m_{u}}$, where $\varepsilon_{i}=0$ or 1 , represent a basis for $U L / I$. Choose the $z_{k}$ to act locally nilpotently in $L$. Then this basis applied to any $\omega \in \wedge^{q} s I$, shows that $\operatorname{polybd}(U L / I) \cdot \omega \leqslant \operatorname{dim} W$. Hence if $u \in \wedge^{q} s L \otimes M$ represents a generator of $N$ then

$$
\operatorname{polybd} N \leqslant \operatorname{polybd}(U L / I \cdot u) \leqslant \operatorname{polybd} M+\operatorname{dim} W .
$$

Substitution in the equation above gives

$$
\begin{aligned}
\operatorname{dim}(L / I)_{\text {even }} & \leqslant \operatorname{grade} N+\operatorname{polybd} M+\operatorname{dim} W \\
& \leqslant \operatorname{grade} M+\operatorname{polybd} M+\operatorname{dim} W .
\end{aligned}
$$

Choose $M$ so that $\operatorname{grade} M+\operatorname{polybd}(M)=\operatorname{polydepth} L$ and choose $I=L_{>2 k}$. Then $V \cong E(L)_{\leqslant 2 k}$ and we have

$$
\operatorname{dim} E(L)_{\leqslant 2 k} \leqslant \text { polydepth } L \text {. }
$$

Since this holds for all $k$ the theorem is proved.

\section{Solvable Lie algebras}

LEMma 3.1. - Let L be a Lie algebra concentrated in odd degrees. Then

$$
\operatorname{Ext}_{U L}(-, U L)=\operatorname{Hom}_{U L}(-, U L) .
$$

In particular

$$
\text { polydepth } L=\operatorname{polybd} U L \text {. }
$$

Proof. - Since $L=L_{\text {odd }}$ it is necessarily abelian. Now $\operatorname{Ext}_{U L}(-, U L)$ is the dual of $\operatorname{Tor}^{U L}\left(-,(U L)^{\#}\right)$ and this is the limit of $\operatorname{Tor}^{U L \leqslant n}\left(-,(U L)^{\#}\right)$, which dualizes to

$$
\operatorname{Ext}_{U L \leqslant n}(-, U L)
$$

Since $U L_{\leqslant n}$ is a finite dimensional exterior algebra and $U L$ is $U L_{\leqslant n}$-free it follows that $\operatorname{Ext}_{U L \leqslant n}^{+}(-, U L)=0$, and so $\operatorname{Ext}_{U L}^{+}(-, U L)=0$.

Finally, since $\operatorname{Ext}_{U L}^{0}(U L, U L)$ is non-zero, polydepth $L \leqslant \operatorname{polybd} U L$. On the other hand if polydepth $L=m<\infty$, then for some $M$, we have

$$
\operatorname{Ext}_{U L}^{p}(M, U L) \neq 0 \text { and } \operatorname{polybd} M=m-p .
$$

By the above, $p=0$ and so there is a non-zero $U L$-linear map $f: M \rightarrow U L$. Any $f(m)$ is in some $U L_{<n}$ and if $f(m) \neq 0$ it follows that $U L_{\geqslant n} \stackrel{\cong}{\longrightarrow} U L_{\geqslant n} \cdot m$. This implies

$$
\operatorname{polybd} M \geqslant \operatorname{polybd} U L \text { and } \operatorname{polydepth} L \geqslant \operatorname{polybd} U L \text {. }
$$


LEMMA 3.2. - Let $L$ be a graded Lie algebra of finite polydepth. If $I$ is an ideal in $L$ and polybd $I<\infty$ then polydepth $L / I<\infty$.

Proof. - Choose $M$ so that polygrade $M=$ polydepth $L$. If $m=\operatorname{grade} M$ then it follows (as in [3], proof of Theorem 4.1 for the case $M=l k$ ) that for some $p$,

$$
\operatorname{Ext}_{U L / I}^{p}\left(\operatorname{Tor}_{m-p}^{U I}(M, l k), U L / I\right) \neq 0 .
$$

Since $\operatorname{Tor}_{m-p}^{U I}(M, l k)$ is a subquotient of $\wedge^{m-p} s I \otimes M$ it follows that it has polynomial growth at most equal to $(m-p)$ polybd $I$.

Proof of Theorem 3. -

(i) $\Rightarrow$ (ii). Let $I$ be the sum of the ideals in $L$ concentrated in odd degrees. Then $I$ is an ideal of this form, necessarily abelian, and $L / I$ has no ideals concentrated in odd degrees. Moreover polybd $U I=\operatorname{polydepth} I \leqslant \operatorname{polydepth} L$ (Lemma 3.1 and Proposition 1.3) and hence polydepth $L / I<\infty$ (Lemma 3.2).

Next we show that every solvable ideal $J$ in $L / I$ is finite dimensional, by induction on the solvlength. Indeed, if $J$ is abelian then $J_{\text {even }}=E(J)$. Since

$$
\text { polydepth } J \leqslant \operatorname{polydepth} L / I \quad \text { (Proposition 1.3), }
$$

Theorem 2 asserts that $J_{\text {even }}$ is finite dimensional. Thus for some $r, J_{\geqslant r}$ is an ideal concentrated in odd degrees, i.e. $J_{\geqslant r}=0$.

Now if $J$ has solvlength $k$ then its $(k+1)$ st derived algebra is abelian and so finite dimensional. Thus for some $r, J_{\geqslant r}$ has solvlength $k-1$. By induction, $J$ is finite dimensional.

By hypothesis $L / I$ is the sum of its solvable ideals. Since these are finite dimensional, each $x \in(L / I)_{\text {even }}$ acts locally nilpotently. Thus $(L / I)_{\text {even }}=E(L / I)$, and this is finite dimensional by Theorem 2 . But $L_{\text {even }} \cong(L / I)_{\text {even }}$ since $I$ is concentrated in odd degrees.

Suppose $L_{\text {even }} \subset L_{\leqslant 2 n}$. Since $L_{>2 n}$ is an ideal in odd degrees of finite polydepth, polybd $U L_{>n}<\infty$, while trivially polybd $U L / L_{>2 n}<\infty$. Hence polybd $U L<\infty$.

(ii) $\Rightarrow$ (iii). Clearly polybd $U L \geqslant \operatorname{dim} L_{\text {even }}$, so the latter must be finite. It is trivial from the Poincaré-Birkhoff-Witt theorem that if $\sum_{i \leqslant n} \operatorname{dim} L_{i}=d(n)$ then

$$
\sum_{i \leqslant n d(n)} \operatorname{dim}(U L)_{i} \geqslant 2^{d(n)}
$$

Thus $2^{d(n)} \leqslant K[n d(n)]^{r}$ for some constant $K$ and some integer $r, r \geqslant 1$. It follows that

$$
d(n) \leqslant \log _{2} K+r \log _{2} n+r \log _{2} d(n) \leqslant r \log _{2} n+\frac{1}{2} d(n),
$$

$n$ sufficiently large.

(iii) $\Rightarrow$ (i). Choose $N$ so that $I=L \geqslant N$ is concentrated in odd degrees. Then $U I$ is an exterior algebra and so

$$
\sum_{i \leqslant n} \operatorname{dim}(U I)_{i} \leqslant 2^{\sum_{i \leqslant n} \operatorname{dim} L_{i}} \leqslant n^{C}
$$

for some constant $C$. Thus, since $L / I$ is finite dimensional, polybd $U L$ is finite. The identity of $U L$ is in $\operatorname{Ext}_{U L}^{0}(U L, U L)$ and so polydepth $L \leqslant \operatorname{polybd} U L$. 
Finally, since $I$ is abelian and $L / I$ is finite dimensional, $L$ itself is solvable. This also proves the last assertion.

Proof of Theorem 4. - This is immediate from Proposition 1.3(i) and Theorem 3.

Proposition 3.3. - Suppose I is a solvable ideal in a Lie algebra L of finite polydepth. Then

(i) polydepth $L / I \leqslant$ polydepth $L$;

(ii) $\operatorname{dim} I_{\text {even }} \leqslant$ polydepth $I \leqslant \operatorname{polybd} U I$.

Proof. - (i) As noted in the proof of Lemma 3.2,

$$
\operatorname{Ext}_{U L / I}^{p}\left(\operatorname{Tor}_{m-p}^{U I}(M, l k), U L / I\right) \neq 0
$$

where $m+\operatorname{polybd} M=$ polydepth $L$. Also the Tor is a subquotient of $\wedge^{m-p} s I \otimes M$. By Theorem 3

$$
\sum_{i \leqslant n} \operatorname{dim}\left(\wedge^{m-p} s I \otimes M\right)_{i} \leqslant\left(C_{1} \log _{2} n\right)^{m-p} \cdot C_{2} \cdot n^{\operatorname{polybd} M}
$$

Hence

$$
\operatorname{polybd} \operatorname{Tor}_{m-p}^{U I}(M, l k) \leqslant \operatorname{polybd} M+1
$$

and so

$$
\text { polydepth } L / I \leqslant \operatorname{polydepth} M+p+1 .
$$

If $p<m$ then this gives polydepth $L / I \leqslant$ polydepth $L$. If $p=m$ then

$$
\operatorname{Tor}_{m-p}^{U I}(M, l k)=M \otimes_{U I} l k .
$$

Hence in this case polybd $\operatorname{Tor}_{m-p}^{U I}(M, l k) \leqslant \operatorname{polybd} M$ and again

$$
\text { polydepth } L / I \leqslant \text { polydepth } L \text {. }
$$

(ii) Since $I_{\text {even }} \subset I_{\leqslant 2 n}$, some $n$ (Theorem 3) we may apply the first assertion to obtain

$$
\begin{aligned}
\operatorname{dim} I_{\text {even }} & =\operatorname{depth} I / I_{>2 n} \quad(\text { cf. [2] }) \\
& =\operatorname{polydepth} I / I_{>2 n} \quad \text { (Proposition 1.6) } \\
& \leqslant \operatorname{polydepth~} I .
\end{aligned}
$$

The second inequality has already been observed:

$$
\operatorname{polybd} U I=\operatorname{polygrade} U I \geqslant \operatorname{polydepth} I .
$$

Example 2. - Consider a Lie algebra $L$ concentrated in odd degrees with a basis $\left\{x_{i}, i \geqslant 1\right\}$ satisfying the degree relations

$$
\operatorname{deg} x_{i}>\sum_{j<i} \operatorname{deg} x_{j}
$$

Then for each $n, \operatorname{dim}(U L)_{n} \leqslant 1$. The identity on $U L$ shows that polydepth $L=1$. 
Example 3.-Consider the graded Lie algebra $L=\mathbb{L}\left(a, x_{n}\right)_{n \geqslant 2} / I$, with $\operatorname{deg} a=2$, $\operatorname{deg} x_{n}=2^{n}+1$, and where $I$ is generated by the relations

$$
\left[(\operatorname{ad} a)^{k} x_{r},(\operatorname{ad} a)^{l} x_{s}\right]=0, \quad k, l \geqslant 0, r, s \geqslant 2, \quad \text { and } \quad \operatorname{ad}^{n+1}(a)\left(x_{n}\right)=0 .
$$

Then polybd $U L=2$, so that $L$ has finite polygrade. On the other hand, $L$ is solvable but not nilpotent, and is the union of the infinite sequence of the finite dimensional Lie algebras $I_{N}$ generated by $a, x_{2}, \ldots, x_{N}$.

Proposition 3.4. - Let $L$ be the direct sum of non-solvable Lie algebras $L(i), i \leqslant n$. If polydepth $L(i)<\infty$ for $1 \leqslant i \leqslant n$, then

$$
n \leqslant \operatorname{polydepth} L \leqslant \sum_{i} \text { polydepth } L(i)
$$

Proof. - We first prove by induction on $n$ that for any $U L$-module $M$ that has at most polynomial growth, we have

$$
\operatorname{Ext}_{U L}^{<n}(M, U L)=0 .
$$

Consider the Hochschild-Serre spectral sequence

$$
\begin{aligned}
& \operatorname{Ext}_{U L(1)}^{p}\left(\operatorname{Tor}_{q}^{U(L(2) \oplus \cdots \oplus L(n))}\left(M,\left(U(L(2) \oplus \cdots \oplus L(n))^{\#}\right), U L(1)\right)\right) \\
& \quad \Rightarrow \operatorname{Ext}_{U L}^{p+q}(M, U L) .
\end{aligned}
$$

Since $L(1)$ commutes with the other $L(i)$ it follows that for each monogenic $U L(1)$-module $N$ that is a subquotient of $\operatorname{Tor}_{q}^{U(L(2) \oplus \cdots \oplus L(n))}\left(M, U(L(2) \oplus \cdots \oplus L(n))^{\#}\right)$ we have

$$
\operatorname{polybd} N \leqslant \operatorname{polybd} M \text {. }
$$

Now since $L(1)$ is not solvable, polybd $U L(1)=\infty$ and the argument in the proof of Lemma 3.1 shows that

$$
\operatorname{Ext}_{U L(1)}^{0}(N, U L(1))=0 .
$$

Thus (Lemma 1.1) the left hand in (4) vanishes for $p=0$. By induction on $n$ it vanishes for $q<n-1$ and so $\operatorname{Ext}_{U L}^{<n}(M, U L)=0$. Thus polydepth $L \geqslant n$.

On the other hand, there are $U L(i)$-modules $M(i)$ such that polygrade $M(i)=\operatorname{polydepth} L(i)$. Then $\bigotimes_{i=1}^{n} M(i)$ is a $U L$-module that has at most polynomial growth and whose polygrade is the sum of the polygrades of the $M(i)$.

\section{Growth of Lie algebras}

PROPOSITION 4.1. - Let L be a non-solvable graded Lie algebra of finite polydepth. Then for each integer $r \geqslant 1$ there is a positive integer $d(r)$ such that

$$
\sum_{i=k+1}^{k+d(r)} \operatorname{dim} L_{i} \geqslant k^{r}, \quad k \text { sufficiently large. }
$$

Proof. - We distinguish two cases.

Case A: $L_{\text {even }}$ contains an infinite dimensional abelian sub Lie algebra $E$. 
Choose $n$ so that $\operatorname{dim} E_{\leqslant n} \geqslant(r+3)$ polydepth $L$. Then there is a finite sequence

$$
L=I(0) \supset I(1) \supset \cdots \supset I(l)
$$

in which $I(j)$ is an ideal in $I(j-1)$ and $I(l)_{\leqslant n}=E_{\leqslant n}$.

By Proposition 1.3, polydepth $I(q) \leqslant$ polydepth $L$. Thus without loss of generality we may suppose that $L=I(l)$, i.e. that $L_{\leqslant n}$ is an abelian sub Lie algebra concentrated in even degrees and that $\operatorname{dim} L_{\leqslant n} \geqslant(r+3)$ polydepth $L$.

Let $M$ be a $U L$-module such that

$$
\text { grade } M+\operatorname{polybd} M=\text { polydepth } L
$$

and put $m=\operatorname{grade} M$. As observed in the proof of Proposition 1(ii),

$$
\operatorname{Ext}_{U L_{\leqslant n}}^{q}\left(\wedge^{p} s L_{>n} \otimes M, U L_{\leqslant n}\right) \neq 0,
$$

for some $p+q=m$. It follows that for some $z \in \wedge^{p} s L_{>n} \otimes M$,

$$
\operatorname{polybd} U L_{\leqslant n} \cdot z+q \geqslant \operatorname{dim} L_{\leqslant n}
$$

(Theorem 3.1 in [3]). Hence for some $x \in L$,

$$
p\left(\operatorname{polybd} U L_{\leqslant n} \cdot x\right) \geqslant \operatorname{dim} L_{\leqslant n}-q-\operatorname{polybd} M .
$$

Since $p+q+\operatorname{polybd} M=$ polydepth $L$ we conclude that

$$
\left(2+\operatorname{polybd} U L_{\leqslant n} \cdot x\right) \cdot \operatorname{polydepth} L \geqslant \operatorname{dim} L_{\leqslant n} \geqslant(r+3) \text { polydepth } L .
$$

As observed in the introduction, since $\operatorname{dim} L=\infty$, polydepth $L>0$. It follows that

$$
\operatorname{polybd}\left(U L_{\leqslant n} \cdot x\right) \geqslant r+1 .
$$

On the other hand, $U L_{\leqslant n}$ is the polynomial algebra $l k\left[y_{1}, \ldots, y_{s}\right]$ on a basis $y_{1}, \ldots, y_{s}$ of $L_{\leqslant n}$. Because of (5) it is easy to see (induction on $s$ ) that this basis can be chosen so that for some $w \in U L_{\leqslant n} \cdot x, l k\left[y_{1}, \ldots, y_{r+1}\right] \rightarrow l k\left[y_{1}, \ldots, y_{r+1}\right] \cdot w$ is injective. Put $d=\prod_{i=1}^{r+1} \operatorname{deg} y_{i}$ and note that

$$
\sum_{i=k+\operatorname{deg} w+1}^{k+\operatorname{deg} w+d} \operatorname{dim} L_{i} \geqslant \sum_{i=k+1}^{k+d} \operatorname{dim} l k\left[y_{1}, \ldots, y_{r+1}\right]_{i} \geqslant \frac{1}{r !} k^{r} .
$$

It follows that for any $r$, and $k$ sufficiently large,

$$
\sum_{i=k+1}^{k+d} \operatorname{dim} L_{i} \geqslant k^{r-1}
$$

This proves Proposition 5 in case A.

Case B: Every abelian sub Lie algebra of $L_{\text {even }}$ is finite dimensional.

Let $I$ be the sum of the solvable ideals in $L$. Then $I_{\text {even }}$ is finite dimensional and polydepth $L / I$ is finite (Theorem 3 and Proposition 3.3). Thus all abelian sub Lie algebras of $L / I$ are finite dimensional. Thus it is sufficient to prove case B when $L$ has no solvable ideals. 
There are now two possibilities: either $L=L_{\text {even }}$, or $L$ has elements of odd degree. In the latter case the sub Lie algebra generated by $L_{\text {odd }}$ is an ideal, hence non-solvable and of finite polydepth. Let $L(s)$ denote the sub Lie algebra generated by the first $s$ linearly independent elements $x_{1}, \ldots, x_{s}$ of odd degree. For $s$ sufficiently large, polydepth $L(s) \leqslant$ polydepth $L$ (Proposition 1) and $\operatorname{dim} L(s)_{\text {even }}>$ polydepth $L$ (obvious). Thus $L(s)$ cannot be solvable (Theorem 3). In other words, we may assume that either $L=L_{\text {even }}$ or else $L$ is generated by finitely many elements $x_{1}, \ldots, x_{s}$ of odd degree. In either case set $E=L_{\text {even }}$, and note that $\operatorname{dim} E$ is infinite.

Define a sequence of elements $z_{i}$ and sub Lie algebras $E(i)$ by setting $E(1)=E, z_{i}$ is a nonzero element in $E(i)$ and $E(i+1) \subset E(i)$ is the sub Lie algebra of elements on which ad $z_{i}$ acts nilpotently.

Since $E$ contains no infinite dimensional abelian Lie algebra some $E(N+1)=0$ and $E(1) / E(2) \oplus \cdots \oplus E(N) / E(N+1)$ is a graded vector space isomorphic with $E$.

Put $d=\prod \operatorname{deg} z_{i}$ and $d_{i}=d / \operatorname{deg} z_{i}$. Then $\left(\operatorname{ad} z_{1}\right)^{d_{1}} \oplus \cdots \oplus\left(\operatorname{ad} z_{N}\right)^{d_{N}}$ is an injective transformation of $E(1) / E(2) \oplus \cdots \oplus E(N) / E(N+1)$ of degree $d$. Since this space is isomorphic with $E$ it follows that

$$
\sum_{i=s}^{t} \operatorname{dim} E_{i} \leqslant \sum_{i=s+d}^{t+d} \operatorname{dim} E_{i} \quad \text { and thus } \sum_{i=k+1}^{k+d} \operatorname{dim} E_{i} \geqslant \frac{d}{k+d} \sum_{i=1}^{k+d} \operatorname{dim} E_{i}, \quad k \geqslant 1 .
$$

On the other hand, choose $n$ so that

$$
\operatorname{dim} E_{\leqslant n} \geqslant(r+3) \cdot \text { polydepth } L
$$

(This is possible because $E$ is infinite dimensional.) Set $I=L_{>n}$. Let $M$ be an $L$-module with polydepth $L=$ polygrade $M$. As in the proof of Theorem 4.1 in [3], $\operatorname{Ext}_{U L / I}^{p}\left(\operatorname{Tor}_{q}^{U I}(M, l k)\right.$, $U L / I) \neq 0$ for $p+q=\operatorname{grade} M$. Thus $p, q$ and $\operatorname{polybd} M$ are all bounded above by polydepth $L$. Now Theorem 3.1 of [3] asserts that for some $\alpha \in \operatorname{Tor}_{q}^{U I}(M, l k), U L / I \cdot \alpha$ has polynomial growth at least equal to $\operatorname{dim}(L / I)_{\text {even }}-p$. This means that for some positive $C$,

$$
\sum_{i \leqslant k} \operatorname{dim}(U L / I \cdot \alpha)_{i} \geqslant C k^{\left(\operatorname{dim}(L / I)_{\text {even }}\right)-p}, \quad k \text { sufficiently large. }
$$

Since $\operatorname{Tor}_{*}^{U I}(M, l k)$ is the homology of $\wedge^{*} s I \otimes M$, it follows that

$$
\sum_{i \leqslant k} \operatorname{dim} \operatorname{Tor}_{q}^{U I}(M, l k)_{i} \leqslant\left(\sum_{i \leqslant k} \operatorname{dim} L_{i}\right)^{q} \sum_{i \leqslant k} \operatorname{dim} M_{i}
$$

But $(L / I)_{\text {even }} \cong E_{\leqslant n}$ and so a quick calculation gives

$$
\sum_{i \leqslant k} \operatorname{dim} L_{i} \geqslant K k^{r+1}, \quad k \text { sufficiently large. }
$$

Finally, recall that either $L=L_{\text {even }}$ or else $L$ is generated by the elements of odd degree $x_{i}$. In the former case $L=E$ and the proposition follows from (6) and (7). In the second case we have

$$
L_{\text {odd }}=\left[x_{1}, E\right]+\cdots+\left[x_{s}, E\right]+l k x_{1}+\cdots+l k x_{s},
$$


and hence (7) yields

$$
\sum_{i \leqslant k} E_{i} \geqslant \frac{K}{s+1} k^{r+1}+s, \quad k \text { sufficiently large. }
$$

Combined with (6) this formula gives the proposition.

Proof of Theorem 5. - Since $L$ is not solvable we may choose $n$ so that

$$
\operatorname{dim}\left(L_{\text {even }}\right) \leqslant n>\text { polydepth } L \quad(\text { Theorem } 3),
$$

and so that the sub Lie algebra generated by $L_{\leqslant n}$ satisfies polydepth $E \leqslant$ polydepth $L$ (Proposition 1.3). Then $E$ is not solvable (Proposition 3.3).

Let $x_{1}, \ldots, x_{s}$ generate $E$ (see beginning of case B) and put $d=\max \operatorname{deg} x_{i}$. Letting $U E$ act via the adjoint representation on $E$ we have that

$$
U E_{[0, q]} \cdot E_{[k+1, k+d]} \supset E_{[k+1, k+q]} \cdot
$$

For any $r \geqslant 1$ choose $q=q(r)$ so that

$$
\sum_{i=k+1}^{k+q} \operatorname{dim} E_{i} \geqslant k^{r+1}
$$

$k$ sufficiently large (Proposition 4.1). Then

$$
\sum_{i=k+1}^{k+d} \operatorname{dim} L_{i} \geqslant \sum_{i=k+1}^{k+d} \operatorname{dim} E_{i} \geqslant \frac{1}{\operatorname{dim} U E_{[0, q]}} k^{r+1} \geqslant k^{r}, \quad k \text { sufficiently large. }
$$

Since $d$ is independent of $r$, the theorem is proved.

\section{REFERENCES}

[1] Felix Y., Halperin S., Lemaire J.-M., Thomas J.-C., Mod p loop space homology, Inventiones math. 95 (1989) 247-262.

[2] Felix Y., Halperin S., Jacobson C., Löfwall C., Thomas J.-C., The radical of the homotopy Lie algebra, Amer. J. Math. 110 (1988) 301-322.

[3] Felix Y., Halperin S., Thomas J.-C., Lie algebras of polynomial growth, J. London Math. Soc. 43 (1991) 556-566.

[4] Felix Y., Halperin S., Thomas J.-C., Hopf algebras of polynomial growth, J. Algebra 125 (1989) 408-417.

[5] Felix Y., Halperin S., Thomas J.-C., Engel elements in the homotopy Lie algebra, J. Algebra 144 (1991) 67-78.

[6] Felix Y., Halperin S., Thomas J.-C., The category of a map and the grade of a module, Israel J. Math. 78 (1992) 177-196.

[7] Felix Y., Halperin S., Thomas J.-C., Growth and Lie brackets in the homotopy Lie algebra, in: The Roos Festschrift, vol. 1, Homology Homotopy Appl. 4, no. 2, part 1, 2002, pp. 219-225.

[8] Halperin S., Universal enveloping algebra and loop space homology, J. Pure Appl. Algebra 83 (1992) 237-282. 
[9] Koszul J.-L., Homologie et cohomologie des algèbres de Lie, Bull. Soc. Math. France 78 (1950) 65-127.

[10] Milnor J.W., Moore J.C., On the structure of Hopf algebras, Ann. Math. 81 (1965) 211-264.

(Manuscrit reçu le 22 juillet 2002 ;

accepté le 24 janvier 2003.)

Yves FELIX

Institut de Mathématiques,

Université Catholique de Louvain,

1348 Louvain-La-Neuve, Belgique

Stephen HALPERIN

College of Computer,

Mathematical and Physical Sciences

University of Maryland,

College Park, MD 20742-3281, USA

Jean-Claude Thomas

Faculté des Sciences,

Université d'Angers,

Bd. Lavoisier,

49045 Angers, France

E-mail: jean-claude.thomas@univ-angers.fr 